Research Paper

\title{
Predictive factors in patients with hepatocellular carcinoma receiving sorafenib therapy using time-dependent receiver operating characteristic analysis
}

\author{
Hiroki Nishikawa ${ }^{{ }^{*}}$, Norihiro Nishijima ${ }^{2 *}$, Hirayuki Enomoto ${ }^{1 凶}$, Azusa Sakamoto $^{2}$, Akihiro Nasu $^{2}$, \\ Hideyuki Komekado ${ }^{2}$, Takashi Nishimura ${ }^{1}$, Ryuichi Kita ${ }^{2}$, Toru Kimura ${ }^{2}$, Hiroko Iijima ${ }^{1}$, Shuhei \\ Nishiguchi ${ }^{1}$, Yukio Osaki ${ }^{2}$
}

1. Division of Hepatobiliary and Pancreatic disease, Department of Internal Medicine, Hyogo College of Medicine, Hyogo, Japan;

2. Department of Gastroenterology and Hepatology, Osaka Red Cross Hospital, Osaka, Japan.

* Hiroki Nishikawa and Norihiro Nishijima contributed equally to this work.

$\square$ Corresponding author: Hirayuki Enomoto, MD, PhD, Department of Hepatobiliary and Pancreatic disease, Department of Internal Medicine, Hyogo College of Medicine, Hyogo, Japan, 1-1, Mukogawacho, Nishinomiyashi, Hyogo, 663-8501, Japan. Tel: +81-798-45-6111; Fax: +81-798-45-6608; Email: enomoto@hyo-med.ac.jp.

(C) Ivyspring International Publisher. This is an open access article distributed under the terms of the Creative Commons Attribution (CC BY-NC) license (https://creativecommons.org/licenses/by-nc/4.0/). See http://ivyspring.com/terms for full terms and conditions.

Received: 2016.07.09; Accepted: 2016.11.10; Published: 2017.02.10

\begin{abstract}
Aims: To investigate variables before sorafenib therapy on the clinical outcomes in hepatocellular carcinoma (HCC) patients receiving sorafenib and to further assess and compare the predictive performance of continuous parameters using time-dependent receiver operating characteristics (ROC) analysis.

Patients and methods: A total of 225 HCC patients were analyzed. We retrospectively examined factors related to overall survival (OS) and progression free survival (PFS) using univariate and multivariate analyses. Subsequently, we performed time-dependent ROC analysis of continuous parameters which were significant in the multivariate analysis in terms of OS and PFS. Total sum of area under the ROC in all time points (defined as TAAT score) in each case was calculated.

Results: Our cohort included 175 male and 50 female patients (median age, 72 years) and included 158 Child-Pugh A and 67 Child-Pugh B patients. The median OS time was 0.68 years, while the median PFS time was 0.24 years. On multivariate analysis, gender, body mass index (BMI), Child-Pugh classification, extrahepatic metastases, tumor burden, aspartate aminotransferase (AST) and alpha-fetoprotein (AFP) were identified as significant predictors of OS and ECOG-performance status, Child-Pugh classification and extrahepatic metastases were identified as significant predictors of PFS. Among three continuous variables (i.e., BMI, AST and AFP), AFP had the highest TAAT score for the entire cohort. In subgroup analyses, AFP had the highest TAAT score except for Child-Pugh B and female among three continuous variables.

Conclusion: In continuous variables, AFP could have higher predictive accuracy for survival in HCC patients undergoing sorafenib therapy.
\end{abstract}

Key words: Hepatocellular carcinoma, Sorafenib, Clinical outcomes, Predictive factor, Time-dependent ROC analysis.

\section{Introduction}

Hepatocellular carcinoma (HCC) is globally one of the leading causes of cancer-related deaths, which accounts for 5-6\% of all new cancers. [1-6] Significant advances in HCC therapy during the last few decades have been achieved. [1, 2, 4, 5] However, unfortunately, a curative treatment for HCC can be 
applied to a limited number of HCC subjects. [5, 7]

Sorafenib is a multi-kinase inhibitory anti-cancer agent that can effectively suppresses tumor growth and cancer cell proliferation. [8, 9] Two pivotal randomized phase III studies presented that subjects with unresectable HCC receiving sorafenib therapy were able to obtain survival benefits as compared with the placebo group with statistical significance. [8, 9] More than half decade have passed after the introduction of sorafenib for unresectable HCC therapy in the clinical settings. However, sorafenib is as yet considered as first-line systemic chemotherapeutic agent for unresectable HCC. [10, 11] Prognostic factors at baseline associated with survival in HCC patients undergoing sorafenib therapy have also been investigated and they include age, gender, liver function, the Eastern Cooperative Oncology Group (ECOG)-performance status (PS), tumor marker and tumor status. [12-21]

Receiver operating characteristics (ROC) analysis is a well-established statistical method and is frequently used to assess the discriminatory ability of continuous parameters for a binary disease outcome such as being alive or dead. However, it should be noted that disease outcomes are usually time-dependent. [22] Time-dependent ROC curves have thus been brought into use to fully evaluate the prediction ability of clinical parameters for time-dependent disease outcomes. [22] However, ROC analysis to evaluate the predictive power of clinical factors for survival that takes time dependence into consideration has not been fully examined in HCC patients undergoing sorafenib therapy. Again, because disease outcomes are often time-dependent, time-dependent ROC analysis seems to be an essential statistical method for the precise evaluation of continuous variables on outcomes.

The aim of the present study was to investigate variables before sorafenib therapy on the clinical outcomes in HCC patients receiving sorafenib. Additionally, in order to further assess and compare the predictive performance of continuous parameters, we created time-dependent ROC curves for censored data and adopted the area under the ROC curve (AUROC) as the criterion. [22]

\section{Patients and methods}

\section{Patients and indications for sorafenib treatment}

Between June 2009 and August 2015, 234 HCC patients treated with sorafenib were admitted to the Division of Hepatobiliary and Pancreatic Disease, Department of Internal Medicine, Hyogo College of Medicine, Hyogo, Japan and at the Department of
Gastroenterology and Hepatology, Osaka Red Cross Hospital, Osaka, Japan. We included the following variables into analysis: age, gender, body mass index (BMI), cause of liver disease, initial dose of sorafenib, Child-Pugh classification, ECOG-PS, HCC stage as defined by Japanese guidelines, laboratory parameters including platelet count, aspartate aminotransferase (AST), alanine aminotransferase (ALT), alkaline phosphatase (ALP), gamma glutamyl transpeptidase (GGT), alpha-fetoprotein (AFP) and des- $\gamma$-carboxy prothrombin (DCP). A total of 225 HCC patients with all these data available were analyzed in this study. HCC was diagnosed as described elsewhere. [23-25] Sorafenib therapy was recommended in patients with unresectable HCCs and with the following clinical features as determined by radiologic findings using dynamic computed tomography (CT): (1) the presence of metastatic lesions outside the liver; (2) poor response to previous transcatheter arterial therapies for HCC (transcatheter arterial chemoembolization [TACE] or transcatheter arterial infusion [TAI] chemotherapy); (3) unsuitability for TACE or TAI due to anatomical reasons; and (4) vascular invasion such as tumor thrombus into the portal vein. [23, 24, 26] Subjects who had poor PS (ECOG-PS $\geq 3$ ) were not recommended for sorafenib therapy. [23, 24]

We retrospectively examined factors related to overall survival (OS) and progression free survival (PFS) using univariate and multivariate analyses. Subsequently, we performed time-dependent ROC analysis of parameters which were significant in the multivariate analysis in terms of OS and PFS. Since this study was a retrospective analysis of patients' clinical data, all treatments were performed in an open-label manner. Our study was performed in accordance with the Declaration of Helsinki and with approvals from the ethics committees of each hospital (Hyogo College of Medicine and Osaka Red Cross Hospital).

\section{Sorafenib therapy}

For subjects with no obvious risk factors, the recommended initial sorafenib dose of $800 \mathrm{mg} /$ day (400 mg twice a day) was administered. [8, 9] In some subjects, the starting dose was reduced considering clinical characteristics such as body weight, age, ECOG-PS, and liver function. The daily sorafenib dose according to the degree of adverse events under sorafenib therapy was adjusted by each attending physician. For subjects who received an initial reduced sorafenib dose with well tolerability, dose escalation was permitted. In cases with adverse events, sorafenib therapy was stopped until the clinical symptoms resolved to grade 2 or less. In 
principle, the treatment efficacy of sorafenib was assessed every 4-8 weeks after the initiation of therapy according to the modified Response Evaluation Criteria in Solid Tumors (mRECIST) and/or the values of tumor markers. [23, 24, 27, 28] Sorafenib therapy was continued until the development of the following conditions: HCC disease progression, unacceptable sorafenib-related drug toxicities, or the patient's wish for stopping treatment. After cessation of sorafenib therapy for any reason, physicians sufficiently evaluated the clinical conditions (tumor status or the general status) of each subject, and evaluated the suitability of other treatments (transcatheter arterial therapies or systemic chemotherapy besides sorafenib) with the purpose of ameliorating clinical outcome. [23-26]

\section{Evaluation of treatment efficacy}

The best treatment efficacy achieved during sorafenib therapy was determined according to the mRECIST criteria and/or tumor marker levels as previously indicated. [23, 24, 27] As reported elsewhere, the treatment efficacy was classified into the following four categories: (i) complete response (CR), (ii) partial response (PR), (iii) stable disease (SD), and (iv) progressive disease (PD). [24, 27] We defined the objective response rate (ORR) as the proportion of subjects with the best tumor treatment response rates considering $\mathrm{CR}$ and PR. We defined the disease control rate (DCR) as the proportion of subjects with the best tumor treatment response rates considering CR, PR, and SD.

\section{Statistical analyses}

In continuous parameters, we performed ROC curve analysis of survival (OS and PFS) for selection of the optimal cutoff value that is associated with maximal total value of specificity and sensitivity and we classified them into two groups using these cutoff points and treated them as categorical covariates. Kaplan-Meier curves were created and compared by using the log-rank test. Parameters with $P$ value less than 0.05 in the univariate analysis were entered into the multivariate analyses (Cox proportional hazard model). Furthermore, we analyzed time-dependent ROC curves of significant predictors for survival (continuous variables) in the multivariate analysis and compared between AUROCs for these parameters in each time point. [22] We also calculated the Total sum of AUROCs in All Time-points (defined as TAAT score) in each continuous parameter. TAAT scores in significant continuous parameters in the multivariate analysis were also compared. In this analysis, we regarded a covariate with higher TAAT score as a covariate with higher predictive power.
OS was defined as the period from the start of sorafenib therapy until death (due to any cause) or the last follow-up visit. PFS was defined as the period from the start of sorafenib therapy until the date of the confirmation of progression-free disease or death (due to any cause). [23, 24] Data were presented as number or median values (range). A $P$ value $<0.05$ was considered statistically significant. All statistical analyses were performed using the JMP 11 software (SAS Institute Inc., Cary, NC).

Table 1. Baseline characteristics $(n=225)$.

\begin{tabular}{|c|c|}
\hline Variables & $\begin{array}{l}\text { Number or } \\
\text { median value (range) }\end{array}$ \\
\hline Age (years) & $72(40-91)$ \\
\hline Gender, male / female & $175 / 50$ \\
\hline Body mass index $\left(\mathrm{kg} / \mathrm{m}^{2}\right)$ & $21.615(14.539-42.192)$ \\
\hline \multicolumn{2}{|l|}{ Causes of liver disease } \\
\hline B / C / non-B and non-C / B and C & $32 / 141 / 48 / 4$ \\
\hline $\begin{array}{l}\text { Initial dose of sorafenib (mg/day), } \\
800 / 600 / 400 / 200\end{array}$ & 64 / 1 / 157 / 3 \\
\hline Child-Pugh classification, A / B & $158 / 67$ \\
\hline \multicolumn{2}{|l|}{ ECOG-performance status } \\
\hline $0 / 1 / 2$ & $191 / 29 / 5$ \\
\hline HCC stage, I / II / III / IVA / IVB & $1 / 17 / 78$ / 44 / 85 \\
\hline \multicolumn{2}{|l|}{ Previous therapies for HCC } \\
\hline Transcatheter arterial therapies, yes / no & 204 / 21 \\
\hline Percutaneous ablative therapies, yes / no & $127 / 98$ \\
\hline Surgical resection, yes / no & $73 / 152$ \\
\hline Tumor burden $\geq 50 \%$, yes / no & $22 / 203$ \\
\hline Total bilirubin (mg/dL) & $0.8(0.2-5.1)$ \\
\hline Serum albumin $(\mathrm{g} / \mathrm{dL})$ & $3.4(1.7-4.8)$ \\
\hline Prothrombin time $(\%)$ & $79.05(48-116)$ \\
\hline Platelet count $\left(\times 10^{4} / \mathrm{mm}^{3}\right)$ & $11.6(3.4-56.7)$ \\
\hline AST (IU/L) & $51(15-791)$ \\
\hline $\operatorname{ALT}(\mathrm{IU} / \mathrm{L})$ & $34(6-380)$ \\
\hline $\operatorname{ALP}(\mathrm{IU} / \mathrm{L})$ & $403(124-4535)$ \\
\hline GGT (IU/L) & $72(14-2172)$ \\
\hline $\operatorname{AFP}(\mathrm{ng} / \mathrm{mL})$ & $141.4(1.7-688400)$ \\
\hline $\mathrm{DCP}(\mathrm{mAU} / \mathrm{mL})$ & 748 (10-421210) \\
\hline \multicolumn{2}{|c|}{$\begin{array}{l}\text { Data are expressed as number or median (range). ECOG; the Eastern Cooperativ } \\
\text { Oncology Group, HCC; hepatocellular carcinoma, AST; aspartate } \\
\text { aminotransferase, ALT; alanine aminotransferase, ALP; alkaline phosphatase, } \\
\text { GGT; gamma glutamyl transpeptidase, AFP; alpha-fetoprotein, DCP; } \\
\text { des- } \gamma \text {-carboxy prothrombin. }\end{array}$} \\
\hline
\end{tabular}

\section{Results}

\section{Baseline characteristics}

The baseline characteristics of analyzed patients $(n=225)$ are presented in Table 1 . They included 175 male and 50 female patients with a median age of 72 years (range, 40-91 years). In terms of cause of liver disease, hepatitis $\mathrm{C}$ virus (HCV) was in the majority. They included 158 patients with Child-Pugh class A and 67 patients with Child-Pugh class B. In 64 (28.4\%) patients, the standard dose of sorafenib $(800 \mathrm{mg} /$ day) was administered at the start of the therapy and 191 patients $(84.9 \%)$ had ECOG-PS 0. The most common previous therapies for HCC included the transcatheter arterial therapies such as TACE or TAI, 
followed by percutaneous ablative therapies, and surgical resection (SR). As for HCC stage, they included stage I in 1 , stage II in 17 , stage III in 78 , stage IVA in 44 and stage IVB in 85, respectively.

\section{OS and PFS in the entire cohort}

The median follow-up period in this study was 0.62 years (0.03-5.58 years). The median OS time was 0.68 years, while the median PFS time was 0.24 years. (Fig. 1A and 1B).

\section{Treatment duration, best treatment response, serious adverse events and causes of death}

The median (range) treatment duration was 0.20 years (0.01-2.94 years). In the analysis of the best tumor response, $\mathrm{CR}$ was achieved in $4, \mathrm{PR}$ in $13, \mathrm{SD}$ in 64, and PD in 88, while 56 were not evaluated (NE); the ORR and DCR were thus calculated to be $7.6 \%$ $(17 / 225)$ and $36.0 \%(81 / 225)$, respectively. Serious adverse events of grade 3 or more as defined by Common Terminology Criteria for Adverse Events (CTCAE) ver. 3.0 were observed in 87 cases $(38.7 \%)$. During the follow-up period, 193 (85.8\%) patients died: 165 due to HCC progression, 7 due to liver failure, and 21 due to other causes.

\section{Univariate and multivariate analyses of parameters contributing to OS}

The univariate analysis identified that the following variables significantly contributed to OS for all cases $(\mathrm{n}=225)$ : gender $(P=0.0121)$; $\mathrm{BMI} \geq 21.94$ $\mathrm{kg} / \mathrm{m}^{2}(P=0.0053)$; initial dose of sorafenib $(P=0.0253)$; ECOG-PS $\quad(P=0.0048) ; \quad$ Child-Pugh classification $(P<0.0001)$; extrahepatic metastases $(P=0.0050)$; portal
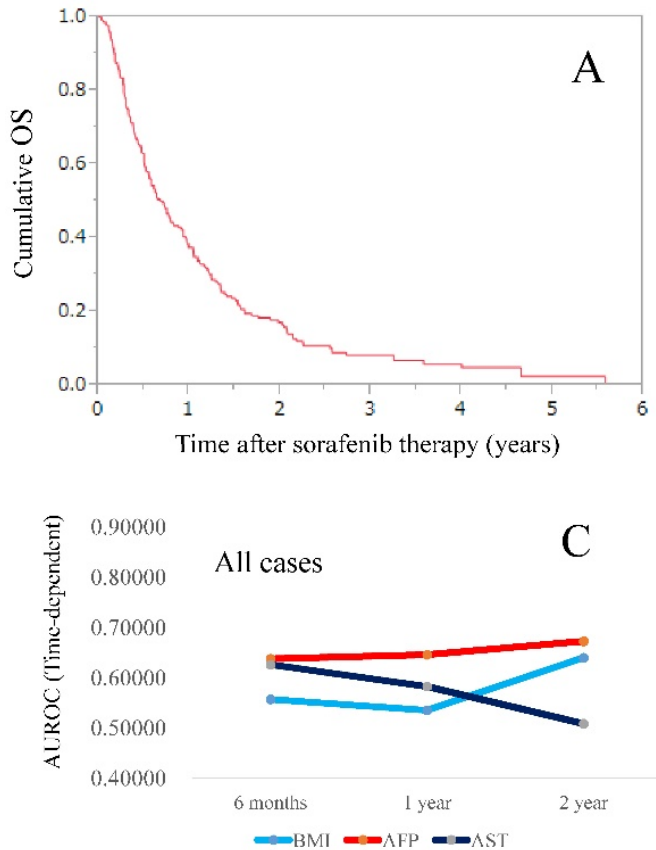

vein invasion $(P=0.0417)$; tumor burden $\geq 50 \%$ $(P=0.0002) ; A S T \geq 24 \mathrm{IU} / 1(P=0.0141) ; A L P \geq 299 \mathrm{IU} / 1$ $(P=0.0368) ;$ GGT $\geq 197 \mathrm{IU} / 1 \quad(P=0.0305) ;$ AFP $\geq 456.5$ $\mathrm{ng} / \mathrm{ml}(P=0.0002)$; and $\mathrm{DCP} \geq 170 \mathrm{mAU} / \mathrm{ml}(P=0.0004)$ (Table 2). The odds ratios (ORs) and $95 \%$ confidence intervals (CIs) as determined by multivariate analysis for the 13 variables (selected based on a $P$ value $<0.05$ in univariate analysis) are listed in table 2. On multivariate analysis, gender $(P=0.0024), \mathrm{BMI} \geq 21.94$ $\mathrm{kg} / \mathrm{m}^{2} \quad(P=0.0035), \quad$ Child-Pugh classification $(P=0.0020)$, extrahepatic metastases $(P=0.0007)$, tumor burden $\geq 50 \% \quad(P=0.0003)$, AST $\geq 29$ IU $/ 1 \quad(P=0.0134)$ and AFP $\geq 456.5 \mathrm{ng} / \mathrm{ml}(P=0.0008)$ were identified as significant predictors of OS.

\section{Univariate and multivariate analyses of factors contributing to PFS}

The univariate analysis identified that the following variables significantly contributed to PFS for all cases $(n=225)$ : ECOG-PS $(P=0.0023)$; Child-Pugh classification $(P=0.0297)$; extrahepatic metastases $(P=0.0027)$; portal vein invasion $(P=0.0313)$; and tumor burden $\geq 50 \%(P=0.0380)$. The ORs and $95 \%$ CIs determined by multivariate analysis for the 5 variables (selected based on a $P$ value $<0.05$ in univariate analysis) are detailed in table 3 . On multivariate analysis, ECOG-PS $(P=0.0188)$, Child-Pugh classification $(P=0.0429)$ and extrahepatic metastases $(P=0.0014)$ were identified as significant predictors of PFS. There were no significant continuous variables linked to PFS in this multivariate analysis. Thus, we did not perform time-dependent ROC analysis of PFS.

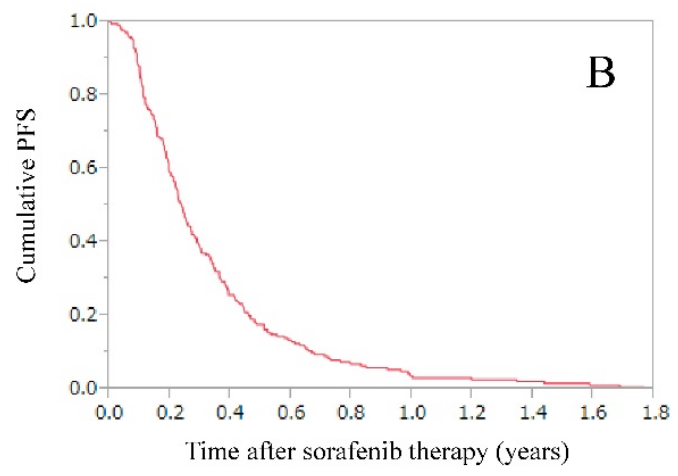

Figure 1. (A) Cumulative overall survival rate for the entire cohort. The median OS time was 0.68 years. (B) Cumulative progression free survival rate for the entire cohort. The median PFS time was 0.24 years. (C) Plots of AUROCs at 6-month, 1-year and 2-year in BMI, AFP and AST values for all cases $(n=225)$. 
Table 2. Univariate and multivariate analyses of factors contributing to overall survival.

\begin{tabular}{|c|c|c|c|c|}
\hline \multirow[t]{2}{*}{ Variables } & \multirow[t]{2}{*}{$\mathrm{n}$} & \multirow{2}{*}{$\begin{array}{l}\text { Univariate } \\
\text { analysis }\end{array}$} & \multicolumn{2}{|l|}{ Multivariate analysis } \\
\hline & & & Odds ratio $(95 \% \mathrm{CI})$ & $P$ value \\
\hline Gender, male vs. female & $175 / 50$ & 0.0121 & $1.885(1.245-2.919)$ & 0.0024 \\
\hline Age (years), $\geq 67$ vs. $<67$ & $165 / 60$ & 0.1671 & & \\
\hline BMI $\left(\mathrm{kg} / \mathrm{m}^{2}\right),<21.94$ vs. $\geq 21.94$ & $120 / 105$ & 0.0053 & $1.563(1.157-2.105)$ & 0.0035 \\
\hline Initial dose of sorafenib, $800 \mathrm{mg} /$ day / reduced dose of sorafenib & $64 / 161$ & 0.0253 & $1.257(0.904-1.770)$ & 0.1766 \\
\hline ECOG-PS 0, yes / no & $191 / 34$ & 0.0048 & $0.784(0.528-1.197)$ & 0.2517 \\
\hline Child-Pugh classification, A / B & $158 / 67$ & $<0.0001$ & $0.581(0.417-0.817)$ & 0.0020 \\
\hline Extrahepatic metastases, yes / no & 85 / 140 & 0.0050 & $1.742(1.267-2.387)$ & 0.0007 \\
\hline Portal vein invasion, yes / no & $50 / 175$ & 0.0417 & $1.313(0.905-1.873)$ & 0.1480 \\
\hline Tumor burden $\geq 50 \%$, yes / no & $22 / 203$ & 0.0002 & $2.841(1.647-4.698)$ & 0.0003 \\
\hline AST (IU/1), $\geq 29$ vs. $<29$ & $191 / 34$ & 0.0141 & $1.778(1.122-2.947)$ & 0.0134 \\
\hline $\operatorname{ALT}(\mathrm{IU} / 1), \geq 54$ vs. $<54$ & $51 / 174$ & 0.6531 & & \\
\hline $\operatorname{ALP}(\mathrm{IU} / 1), \geq 299$ vs. $<299$ & $174 / 51$ & 0.0368 & $1.015(0.698-1.507)$ & 0.9409 \\
\hline GGT (IU/1), $\geq 197$ vs. $<197$ & $40 / 185$ & 0.0305 & $1.203(0.696-1.534)$ & 0.9110 \\
\hline Platelet count $\left(\times 10^{4} / \mathrm{mm}^{3}\right), \geq 16.7$ vs. $<16.7$ & $59 / 166$ & 0.1057 & & \\
\hline Serum AFP $(\mathrm{ng} / \mathrm{ml}), \geq 456.5$ vs. $<456.5$ & $88 / 137$ & 0.0002 & $1.758(1.269-2.424)$ & 0.0008 \\
\hline $\mathrm{DCP}(\mathrm{mAU} / \mathrm{ml}), \geq 170$ vs. $<170$ & $158 / 67$ & 0.0004 & $1.098(0.769-1.587)$ & 0.6109 \\
\hline
\end{tabular}

CI; confidence interval, BMI; body mass index, ECOG-PS; the Eastern Cooperative Oncology Group performance status, AST; aspartate aminotransferase, ALT; alanine aminotransferase, ALP; alkaline phosphatase, GGT; gamma glutamyl transpeptidase, AFP, alpha-fetoprotein; DCP, des- $\gamma$-carboxy prothrombin, a; Cox proportional hazard model.

Table 3. Univariate and multivariate analyses of factors contributing to progression free survival.

\begin{tabular}{|c|c|c|c|c|}
\hline \multirow[t]{2}{*}{ Variables } & \multirow[t]{2}{*}{$\mathrm{n}$} & \multirow{2}{*}{$\begin{array}{l}\text { Univariate } \\
\text { analysis }\end{array}$} & \multicolumn{2}{|l|}{ Multivariate analysis } \\
\hline & & & Odds ratio $(95 \% \mathrm{CI})$ & $P$ value \\
\hline Gender, male vs. female & $175 / 50$ & 0.3035 & & \\
\hline Age (years), $\geq 60$ vs. $<60$ & $196 / 29$ & 0.0878 & & \\
\hline BMI $\left(\mathrm{kg} / \mathrm{m}^{2}\right), \geq 22.05$ vs. $<22.05$ & $101 / 124$ & 0.9923 & & \\
\hline Initial dose of sorafenib, $800 \mathrm{mg} /$ day / reduced dose of sorafenib & $64 / 161$ & 0.1030 & & \\
\hline ECOG-PS 0, yes / no & $191 / 34$ & 0.0023 & $0.614(0.424-0.919)$ & 0.0188 \\
\hline Child-Pugh classification, A / B & $158 / 67$ & 0.0297 & $0.725(0.536-0.990)$ & 0.0429 \\
\hline Extrahepatic metastases, yes / no & 85 / 140 & 0.0027 & $1.611(1.206-2.141)$ & 0.0014 \\
\hline Portal vein invasion, yes / no & $50 / 175$ & 0.0313 & $1.330(0.939-1.854)$ & 0.1067 \\
\hline Tumor burden $\geq 50 \%$, yes / no & $22 / 203$ & 0.0380 & $1.493(0.921-2.303)$ & 0.1004 \\
\hline AST (IU/l), $\geq 28$ vs. $<28$ & $198 / 27$ & 0.4771 & & \\
\hline $\operatorname{ALT}(\mathrm{IU} / \mathrm{l}), \geq 19$ vs. $<19$ & 194 / 31 & 0.5663 & & \\
\hline $\operatorname{ALP}(\mathrm{IU} / \mathrm{l}), \geq 1045$ vs. $<1045$ & $15 / 210$ & 0.2215 & & \\
\hline GGT (IU/1), $\geq 310$ vs. $<310$ & $23 / 202$ & 0.7722 & & \\
\hline Platelet count $\left(\times 10^{4} / \mathrm{mm}^{3}\right), \geq 14.4$ vs. $<14.4$ & $82 / 143$ & 0.0756 & & \\
\hline Serum AFP $(\mathrm{ng} / \mathrm{ml}), \geq 17.5$ vs. $<17.5$ & 164 / 61 & 0.3883 & & \\
\hline $\mathrm{DCP}(\mathrm{mAU} / \mathrm{ml}), \geq 3360$ vs. $<3360$ & $74 / 151$ & 0.1945 & & \\
\hline
\end{tabular}

CI; confidence interval, BMI; body mass index, ECOG-PS; the Eastern Cooperative Oncology Group performance status, AST; aspartate aminotransferase, ALT; alanine aminotransferase, ALP; alkaline phosphatase, GGT; gamma glutamyl transpeptidase, AFP, alpha-fetoprotein; DCP, des- $\gamma$-carboxy prothrombin, a; Cox proportional hazard model.

\section{Time-dependent ROC analysis of BMI, AFP and AST values for OS in the entire cohort}

Plots of AUROCs (at 6-month, 1-year and 2-year) of BMI, AFP and AST for OS in the entire cohort are shown in table 4 and fig. 1C. BMI, AFP and AST are significant continuous parameters in our multivariate analysis for OS. The AUROCs at 6-month, 1- and 2 -year were $0.55693,0.53517$ and 0.63980 , respectively, in BMI, 0.63766, 0.64577 and 0.67266, respectively, in AFP and $0.62600,0.58252$ and 0.50846 , respectively, in AST. The TAAT score in AFP was the highest among three continuous parameters (TAAT score=1.95609).

\section{Time-dependent ROC analysis of BMI, AFP and AST values for OS according to Child-Pugh classification}

In patients with Child-Pugh A $(\mathrm{n}=158)$, the
TAAT score in AFP (1.90306) was the highest, followed by that in BMI (1.75549). (Fig. 2A) In patients with Child-Pugh B $(n=67)$, the TAAT score in AST (1.98912) was the highest, followed by that in AFP (1.78791). (Fig. 2B)

\section{Time-dependent ROC analysis of BMI, AFP and AST values for OS according to ECOG-PS}

In patients with ECOG-PS $0(n=191)$, the TAAT score in AFP (1.86815) was the highest, followed by that in AST (1.71484). (Fig. 2C) In patients with ECOG-PS 1 or $2(n=34)$, the TAAT score in AFP (2.26035) was the highest, followed by that in BMI (2.05597). (Fig. 2D) 
A

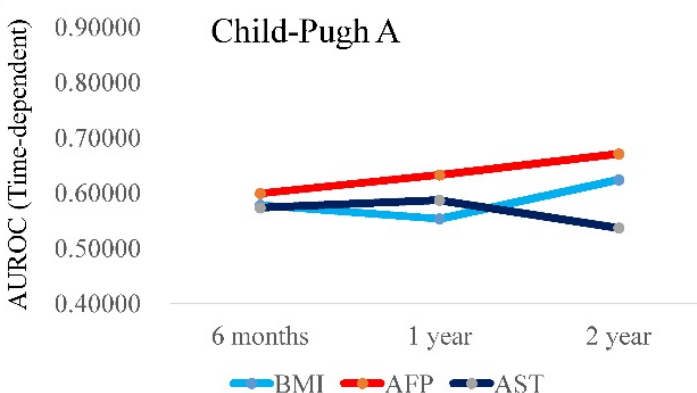

C

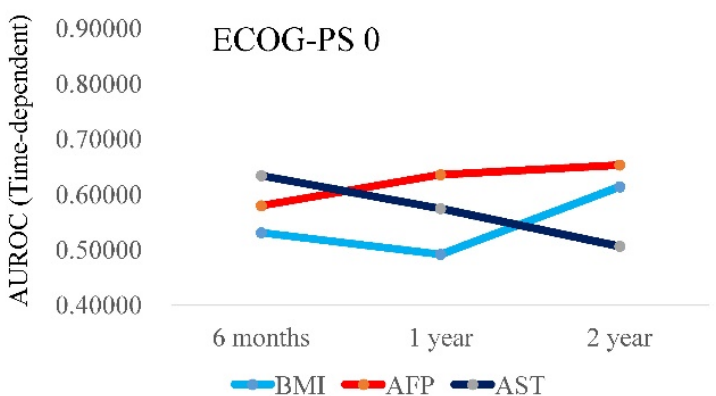

B

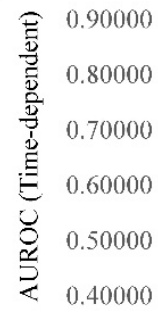

Child-Pugh B

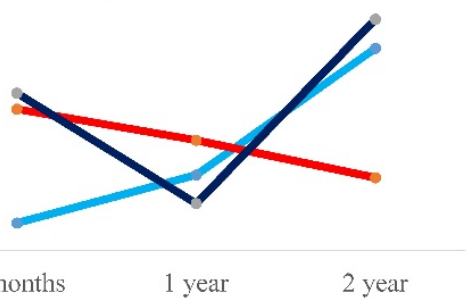

$\rightarrow$ BMI $=$ AFP $=$ AST

$\mathrm{D}$

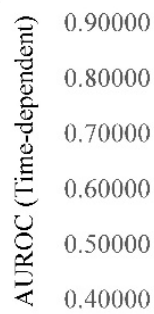

ECOG-PS 1 or 2

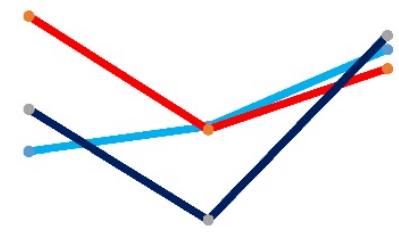

$$
6 \text { months } \quad 1 \text { year } \quad 2 \text { year }
$$$$
=\mathrm{BMI}=\mathrm{ATP}=\mathrm{AST}
$$

Figure 2. (A and B) Plots of AUROCs at 6-month, 1-year and 2-year in BMI, AFP and AST values for patients with Child-Pugh A (2A, $n=158)$ and Child-Pugh B (2B, $\mathrm{n}=67)$. (C and D) Plots of AUROCs at 6-month, 1-year and 2-year in BMI, AFP and AST values for patients with ECOG-PS 0 (2C, $n=191)$ and ECOG-PS 1 or 2 (2D, $\mathrm{n}=34)$.

Table 4. Time-dependent ROC analysis among three continuous parameters that were significant in the multivariate analysis.

\begin{tabular}{|c|c|c|c|c|}
\hline All cases $(n=225)$ & 6-month & 1-year & 2-year & TAAT score \\
\hline BMI & 0.55693 & 0.53517 & 0.63980 & 1.73190 \\
\hline AFP & 0.63766 & 0.64577 & 0.67266 & 1.95609 \\
\hline AST & 0.62600 & 0.58252 & 0.50846 & 1.71698 \\
\hline Child-Pugh A (n=158) & 6-month & 1-year & 2-year & TAAT score \\
\hline BMI & 0.57797 & 0.55342 & 0.62410 & 1.75549 \\
\hline AFP & 0.59943 & 0.63283 & 0.67080 & 1.90306 \\
\hline AST & 0.57323 & 0.58700 & 0.53682 & 1.69705 \\
\hline Child-Pugh B (n=67) & 6-month & 1-year & 2-year & TAAT score \\
\hline BMI & 0.45009 & 0.53676 & 0.76608 & 1.75293 \\
\hline AFP & 0.65608 & 0.59967 & 0.53216 & 1.78791 \\
\hline AST & 0.68512 & 0.48529 & 0.81871 & 1.98912 \\
\hline ECOG-PS 0 (n=191) & 6-month & 1-year & 2-year & TAAT score \\
\hline BMI & 0.53084 & 0.49166 & 0.61376 & 1.63626 \\
\hline AFP & 0.57988 & 0.63535 & 0.65292 & 1.86815 \\
\hline AST & 0.63376 & 0.57469 & 0.50639 & 1.71484 \\
\hline ECOG-PS 1 or $2(n=34)$ & 6-month & 1-year & 2-year & TAAT score \\
\hline BMI & 0.60902 & 0.65385 & 0.79310 & 2.05597 \\
\hline AFP & 0.85338 & 0.64835 & 0.75862 & 2.26035 \\
\hline AST & 0.68512 & 0.48529 & 0.81871 & 1.98912 \\
\hline Tumor burden <50\% $(n=203)$ & 6-month & 1-year & 2-year & TAAT score \\
\hline BMI & 0.55116 & 0.54275 & 0.64580 & 1.73971 \\
\hline AFP & 0.63387 & 0.64325 & 0.68037 & 1.95749 \\
\hline AST & 0.56443 & 0.55267 & 0.53042 & 1.64752 \\
\hline Extrahepatic metastasis, yes $(\mathrm{n}=85)$ & 6-month & 1-year & 2-year & TAAT score \\
\hline BMI & 0.56316 & 0.49153 & 0.47418 & 1.52887 \\
\hline AFP & 0.67685 & 0.64972 & 0.70423 & 2.03080 \\
\hline AST & 0.37662 & 0.42090 & 0.54460 & 1.34212 \\
\hline Extrahepatic metastasis, no $(n=140)$ & 6-month & 1-year & 2-year & TAAT score \\
\hline BMI & 0.55629 & 0.54864 & 0.68067 & 1.78560 \\
\hline AFP & 0.60769 & 0.63906 & 0.65523 & 1.90198 \\
\hline AST & 0.64432 & 0.60034 & 0.53782 & 1.78248 \\
\hline Portal vein invasion, yes $(n=50)$ & 6-month & 1-year & 2-year & TAAT score \\
\hline BMI & 0.61376 & 0.68891 & 0.81220 & 2.11487 \\
\hline
\end{tabular}




\begin{tabular}{|c|c|c|c|c|}
\hline AFP & 0.69136 & 0.67647 & 0.82927 & 2.19710 \\
\hline AST & 0.62522 & 0.53054 & 0.55122 & 1.70698 \\
\hline Portal vein invasion, no $(n=175)$ & 6-month & 1-year & 2-year & TAAT score \\
\hline BMI & 0.48793 & 0.50715 & 0.58850 & 1.58358 \\
\hline AFP & 0.64255 & 0.63356 & 0.62431 & 1.90042 \\
\hline AST & 0.62006 & 0.59724 & 0.50207 & 1.71937 \\
\hline Male $(n=175)$ & 6-month & 1-year & 2-year & TAAT score \\
\hline BMI & 0.59170 & 0.54334 & 0.59903 & 1.73407 \\
\hline AFP & 0.65639 & 0.67630 & 0.81380 & 2.14649 \\
\hline AST & 0.62553 & 0.57037 & 0.51968 & 1.71558 \\
\hline Female $(n=50)$ & 6-month & 1-year & 2-year & TAAT score \\
\hline BMI & 0.58225 & 0.50000 & 0.71765 & 1.79990 \\
\hline AFP & 0.59740 & 0.54808 & 0.52509 & 1.67057 \\
\hline AST & 0.57576 & 0.62714 & 0.45882 & 1.66172 \\
\hline
\end{tabular}

ECOG-PS; the Eastern Cooperative Oncology Group performance status, BMI; body mass index, AFP; alpha-fetoprotein, AST; aspartate aminotransferase. TAAT score indicates Total sum of AUROCs in All Time-points.

A

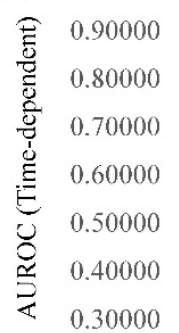

Extrahepatic metastasis $(+)$

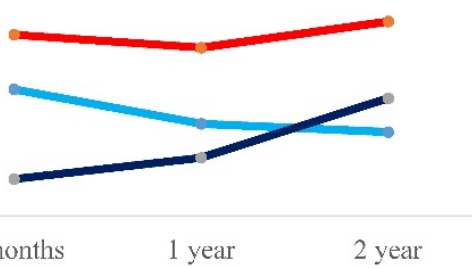

$\Leftrightarrow \mathrm{BMI}=\mathrm{APP}=\mathrm{ASI}$

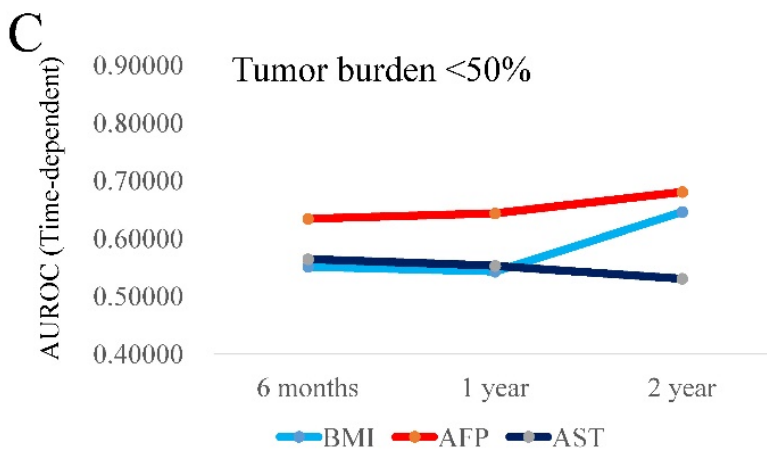

$\mathrm{B}$

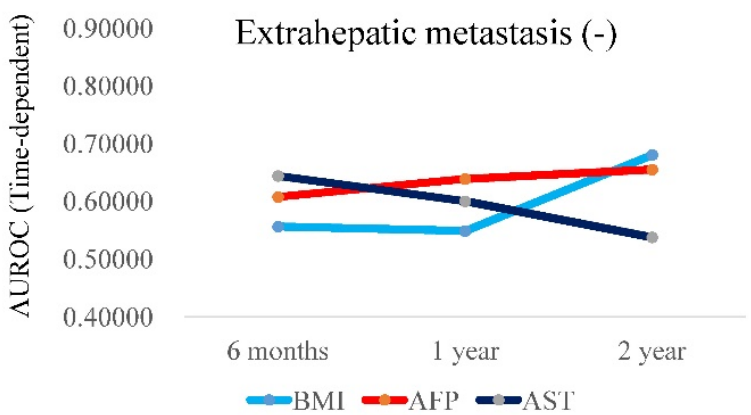

Figure 3. (A and B) Plots of AUROCs at 6-month, 1-year and 2-year in BMI, AFP and AST values for patients with extrahepatic metastases (3A, $n=85)$ and without extrahepatic metastases $(3 \mathrm{~B}, \mathrm{n}=140)$. (C) Plots of AUROCs at 6 -month, 1 -year and 2 -year in $B M I, A F P$ and AST values for patients with tumor burden $<50 \%(3 C$, $\mathrm{n}=203)$.

\section{Time-dependent ROC analysis of BMI, AFP and AST values for OS according to the tumor status (extrahepatic metastasis, tumor burden or portal vein invasion)}

In patients with extrahepatic metastasis $(n=85)$, the TAAT score in AFP (2.03080) was the highest, followed by that in BMI (1.52887). (Fig. 3A) In patients without extrahepatic metastasis $(n=140)$, the TAAT score in AFP (1.90198) was the highest, followed by that in BMI (1.78560). (Fig. 3B). In patients with tumor burden $<50 \% \quad(n=203)$, the TAAT score in AFP (1.95749) was the highest, followed by that in BMI (1.73971). (Fig. 3C) Due to the small number of cases in patients with tumor burden $\geq 50 \% \quad(n=22)$, we did not perform time-dependent ROC analysis in this cohort. In patients with portal vein invasion $(n=50)$, the TAAT score in AFP (2.19710) was the highest, followed by that in BMI (1.52887). (Fig. 4A) In patients without portal vein invasion $(\mathrm{n}=175)$, the TAAT score in AFP (1.90042) was the highest, followed by that in AST (1.71937). (Fig. 4B).

\section{Time-dependent ROC analysis of BMI, AFP and AST values for OS according to gender}

In male patients $(\mathrm{n}=175)$, the TAAT score in AFP (2.14649) was the highest, followed by that in BMI (1.73407). (Fig. 4C) In female patients $(n=50)$, the TAAT score in BMI (1.79990) was the highest, followed by that in AFP (1.67057). (Fig. 4D) 
A

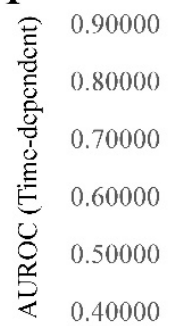

Portal vein invasion $(+)$

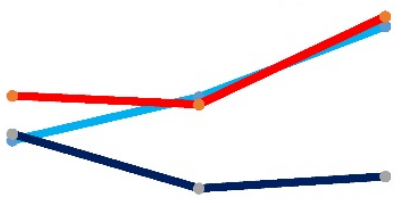

6 months 1 year 2 year

- BMI $=$ AFP $=-$ AST

C

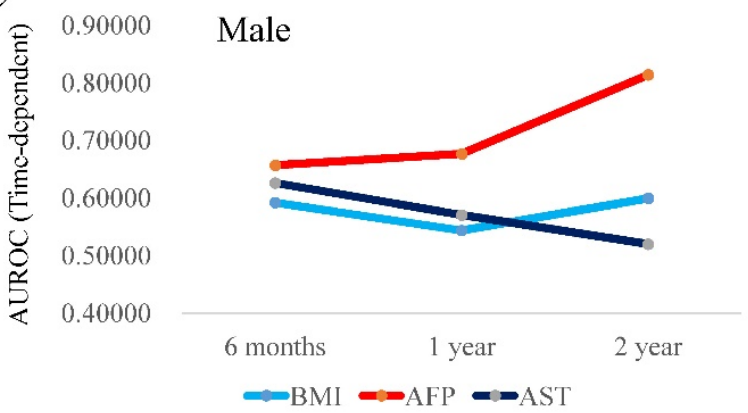

$\mathrm{B}$

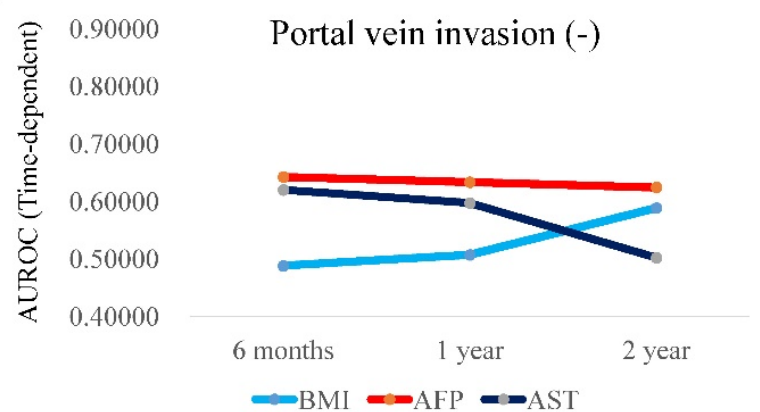

$\mathrm{D}$

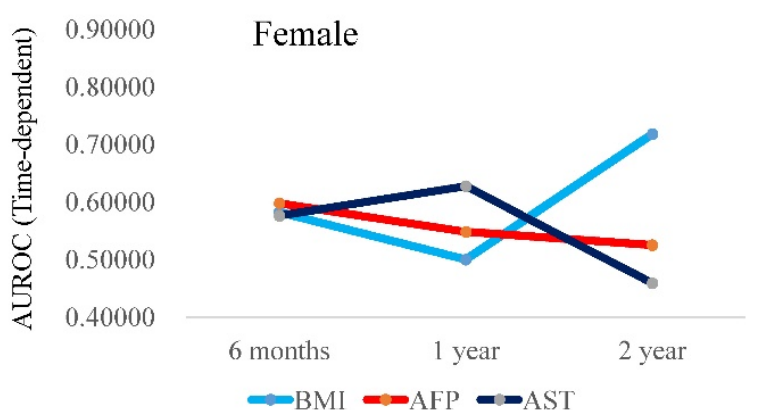

Figure 4. (A and B) Plots of AUROCs at 6-month, 1 -year and 2-year in BMI, AFP and AST values for patients with portal vein invasion (4A, $n=50)$ and without portal vein invasion (4B, $n=175)$. ( $C$ and $D)$ Plots of AUROCs at 6-month, 1-year and 2-year in BMI, AFP and AST values for male patients (4C, $n=175)$ and female patients $(4 \mathrm{D}, \mathrm{n}=50)$.

\section{Discussion}

To the best of our knowledge, there have been no reports with regard to prognostic investigation for HCC patients receiving sorafenib therapy using time-dependent ROC analysis. As mentioned earlier, it should be highlighted that most clinical continuous parameters are time-dependent. Investigation using time-dependent ROC analysis is clinically beneficial. We therefore conducted this current analysis. The major advantage of this study is the large number of enrolled subjects. Our proposed TAAT scoring system is the novel scoring system. As demonstrated in our figures, lines with plots of AUROCs in continuous parameters tended to be crossed. We therefore adopted this scoring system for assessing the predictive ability of outcomes. The shorter median OS time in this study (0.68 years) compared with the previous report can be explained by the higher proportion of Child-Pugh B patients in our data (29.8\% [67/225]). [8]

In our time-dependent ROC analyses, AFP had the highest TAAT score among three continuous parameters and in many of sub-group analyses, similar trends were confirmed. These results indicate that in continuous variables, AFP had the highest predictive ability for survival in HCC patients undergoing sorafenib therapy. Because AFP is a well verified predictive factor in HCC patients receiving sorafenib therapy, it is not so surprising to obtain current results. [12-21] However, these have not been confirmed using time-dependent analysis. In this regard, we believe that our current results are worth reporting.

On the other hand, in terms of ORs in BMI, AST and AFP, AST had the highest OR (1.778) for OS. However, the TAAT score of AFP was the highest for the entire cohort. The effect of continuous variables on clinical outcome should not be determined based on OR alone. It is also of note that the TAAT score of AFP was the highest among three significant continuous parameters irrespective of tumor status (extrahepatic metastasis or portal vein invasion). These results may shed some lights on the sorafenib therapy in HCC.

Lower BMI was an adverse predictor in our results. Previous studies demonstrated that higher BMI was an adverse predictor in HCC patients undergoing TACE, while it was a favorable predictor in HCC patients undergoing SR. [29, 30] On the other hand, in our previous studies, BMI itself did not affect prognosis in HCV-related or non-B and non-C HCC patients undergoing SR. [31, 32] Thus, whether BMI can affect prognosis in HCC patients remains unclear. However, higher prevalence of poorer ECOG-PS (PS $\geq 1)$ in patients with BMI $<21.94 \mathrm{~kg} / \mathrm{m}^{2}(19.17 \%$, [23/120]) as compared with that in patients with BMI 
$\geq 21.94 \mathrm{~kg} / \mathrm{m}^{2}(10.48 \%,[11 / 105])$ may explain our current results.

In our data, female patients had significantly longer OS time than male patients and it revealed to be significant in the multivariate analysis in terms of OS. Our previous study demonstrated that female gender was an independent predictor of good response to sorafenib. [23] This may be associated with our current results.

We acknowledge several limitations of the present study. Firstly, this is a retrospective observational study. Secondly, the initial dose of sorafenib differed between the patients and the duration of sorafenib administration in this study is relatively short, creating bias. Thirdly, various anticancer therapies were employed after the cessation of sorafenib therapy, and these therapies could have potentially caused bias in the clinical outcomes of the analyzed subjects. Fourthly, our study population only included Japanese patients with relatively low body weights compared to patients in Western countries: whether our results can be applied to HCC patients with different patient backgrounds remains unknown. Fifthly, the data of period from initial HCC diagnosis until death are missing, also creating bias. Finally, our proposed TAAT scoring system is not a well-established statistical method and it should be fully verified in the future. However, in this study, AFP value was confirmed to be a highly useful predictor in HCC patients receiving sorafenib therapy using time-dependent ROC analysis.

In conclusion, we examined prognostic parameters in HCC patients treated with sorafenib using time-dependent ROC analysis. Among continuous variables, AFP could have the highest predictive ability for survival.

\section{Abbreviations}

HCC: hepatocellular carcinoma; ECOG-PS: the Eastern Cooperative Oncology Group-performance status; ROC: receiver operating characteristics; AUROC: area under the ROC; BMI: body mass index; AST: aspartate aminotransferase; ALT: alanine aminotransferase; ALP: alkaline phosphatase; GGT: gamma glutamyl transpeptidase; AFP: alpha-fetoprotein; DCP: des- $\gamma$-carboxy prothrombin; CT: dynamic computed tomography; TACE: transcatheter arterial chemoembolization; TAI: transcatheter arterial infusion chemotherapy; OS: overall survival; PFS: progression free survival; mRECIST: modified Response Evaluation Criteria in Solid Tumors; CR: complete response; PR: partial response; SD: stable disease; $\mathrm{PD}$ : progressive disease; ORR: objective response rate; DCR: disease control rate; TAAT score: Total sum of AUROCs in All Time-points; HCV: hepatitis C virus; SR: surgical resection; CTCAE: Common Terminology Criteria for Adverse Events; NE: not evaluated; OR: odds ratio; CI: confidence interval.

\section{Acknowledgements}

The authors would like to thank Haruko Takada (Osaka Red Cross Hospital), Nozomi Kanazawa (Hyogo College of Medicine), Yoko Matsushita (Hyogo College of Medicine), and Sayaka Fujii (Hyogo College of Medicine) for data collection.

\section{Competing Interests}

The authors have declared that no competing interest exists.

\section{References}

[1] Singal AG, El-Serag HB. Hepatocellular Carcinoma From Epidemiology to Prevention: Translating Knowledge into Practice. Clin Gastroenterol Hepatol 2015; 13(12): 2140-2151.

[2] McGlynn KA, Petrick JL, London WT. Global epidemiology of hepatocellular carcinoma: an emphasis on demographic and regional variability. Clin Liver Dis 2015; 19(2): 223-238.

[3] El-Serag HB. Hepatocellular carcinoma. N Engl J Med 2011; 365: 1118-1127.

[4] El-Serag HB. Epidemiology of viral hepatitis and hepatocellular carcinoma. Gastroenterology 2012; 142: 1264-1273.

[5] Osaki Y, Nishikawa H. Treatment for hepatocellular carcinoma in Japan over the last three decades: Our experience and literature review. Hepatol Res 2014 Jun 26. [Epub ahead of print]

[6] Jemal A, Bray F, Center MM, Ferlay J, Ward E, Forman D. Global cancer statistics. CA Cancer J Clin. 2011; 61(2): 69-90.

[7] Rahbari NN, Mehrabi A, Mollberg NM, et al. epatocellular carcinoma: current management and perspectives for the future. Ann Surg 2011; 253(3): 453-469.

[8] Llovet JM, Ricci S, Mazzaferro V, et al. SHARP Investigators Study Group: Sorafenib in advanced hepatocellular carcinoma. N Engl J Med 2008; 359: 378-390.

[9] Cheng AL, Kang YK, Chen Z, et al. Efficacy and safety of sorafenib in patients in the Asia-Pacific region with advanced hepatocellular carcinoma: a phase III randomised, double-blind, placebo-controlled trial. Lancet Oncol 2009; 10: 25-34.

[10] Sangiovanni A, Colombo M. Treatment of hepatocellular carcinoma: beyond international guidelines. Liver Int 2016; 36 Suppl 1: 124-129.

[11] Bruix J, Reig M, Sherman M. Evidence-Based Diagnosis, Staging, and Treatment of Patients With Hepatocellular Carcinoma. Gastroenterology. 2016; 150(4): 835-853

[12] Bruix J, Raoul JL, Sherman M, et al. Efficacy and safety of sorafenib in patients with advanced hepatocellular carcinoma: subanalyses of a phase III trial. J Hepatol 2012; 57(4): 821-829.

[13] Llovet JM, Peña CE, Lathia CD, Shan M, Meinhardt G, Bruix J. SHARP Investigators Study Group. Plasma biomarkers as predictors of outcome in patients with advanced hepatocellular carcinoma. Clin Cancer Res 2012; 18(8): 2290-2300.

[14] Ogasawara S, Chiba T, Ooka Y, et al. Liver function assessment according to the Albumin-Bilirubin (ALBI) grade in sorafenib-treated patients with advanced hepatocellular carcinoma. Invest New Drugs 2015 Oct 14. [Epub ahead of print]

[15] Choi GH, Shim JH, Kim MJ, et al Sorafenib alone versus sorafenib combined with transarterial chemoembolization for advanced-stage hepatocellular carcinoma: results of propensity score analyses. Radiology 2013; 269(2): 603-611.

[16] Kudo M, Matsui O, Izumi N, et al. Liver Cancer Study Group of Japan. Transarterial chemoembolization failure/refractoriness: JSH-LCSGJ criteria 2014 update. Oncology 2014; 87 Suppl 1: 22-31.

[17] Printz C. Clinical trials of note. Sorafenib as adjuvant treatment in the prevention of disease recurrence in patients with hepatocellular carcinoma (HCC) (STORM). Cancer 2009; 115(20): 4646.

[18] Takeda H, Nishikawa H, Osaki Y, et al; Japanese Red Cross Liver Study Group. Proposal of Japan Red Cross score for sorafenib therapy in hepatocellular carcinoma. Hepatol Res. 2015; 45(10): E130-140.

[19] Kaneko S, Ikeda K, Matsuzaki Y, et al. Safety and effectiveness of sorafenib in Japanese patients with hepatocellular carcinoma in daily medical practice: interim analysis of a prospective postmarketing all-patient surveillance study. J Gastroenterol. 2016 Mar 1. [Epub ahead of print] 
[20] Imanaka K, Ohkawa K, Tatsumi T, et al. Impact of branched-chain amino acid supplementation on the survival in patients with advanced hepatocellular carcinoma treated with sorafenib; a multicenter retrospective cohort study. Hepatol Res. 2015 Dec 22. [Epub ahead of print]

[21] Ogasawara S, Chiba T, Ooka Y, et al. Sorafenib treatment in Child-Pugh A and B patients with advanced hepatocellular carcinoma: safety, efficacy and prognostic factors. Invest New Drugs. 2015; 33(3): 729-739.

[22] Heagerty PJ, Zheng Y. Survival model predictive accuracy and ROC curves. Biometrics 2005; 61: 92-105.

[23] Takeda H, Nishikawa H, Osaki Y, et al. Japanese Red Cross Liver Study Group: Clinical features associated with radiological response to sorafenib in unresectable hepatocellular carcinoma: a large multicenter study in Japan. Liver Int 2014 May 16. [Epub ahead of print]

[24] Nishikawa H, Takeda H, Tsuchiya $K$, et al. Japanese Red Cross Liver Study Group: Sorafenib therapy for BCLC stage B/C hepatocellular carcinoma; clinical outcome and safety in aged patients: A multicenter study in Japan. J. Cancer 2014; 5(7): 499-509.

[25] European Association For The Study Of The Liver; European Organisation For Research And Treatment Of Cancer: EASL-EORTC Clinical Practice guidelines: Management of hepatocellular carcinoma. J Hepatol 2012; 56: 908-943.

[26] Cheng AL, Amarapurkar D, Chao Y, et al. Re-evaluating transarterial chemoembolization for the treatment of hepatocellular carcinoma: Consensus recommendations and review by an International Expert Panel. Liver Int 2014; 34(2): 174-183.

[27] Lencioni R, Llovet JM. Modified RECIST (mRECIST) assessment for hepatocellular carcinoma. Seminars in Liver Disease 2010; 30: 52-60.

[28] Salvaggio G, Furlan A, Agnello F, et al. Hepatocellular carcinoma enhancement on contrast-enhanced CT and MR imaging: response assessment after treatment with sorafenib: preliminary results. Radiol Med 2014; 119(4): 215-221.

[29] Wu SE, Charles HW, Park JS, Goldenberg AS, Deipolyi AR. Obesity conveys poor outcome in patients with hepatocellular carcinoma treated by transarterial chemoembolization. Diagn Interv Imaging. 2016 Jun 29. [Epub ahead of print]

[30] Lee YL, Li WC, Tsai TH, Chiang HY, Ting CT. Body mass index and cholesterol level predict surgical outcome in patients with hepatocellular carcinoma in taiwan - a cohort study. Oncotarget. 2016 Mar 24. [Epub ahead of print]

[31] Nishikawa H, Osaki $Y$, Takeda $\mathrm{H}$, et al. Effect of body mass index on survival after curative therapy for non-B non-C hepatocellular carcinoma. J Gastrointestin Liver Dis. 2013; 22(2): 173-181.

[32] Nishikawa H, Arimoto A, Wakasa T, Kita R, Kimura T, Osaki Y. The Relation between Obesity and Survival after Surgical Resection of Hepatitis C Virus-Related Hepatocellular Carcinoma. Gastroenterol Res Pract. 2013; 2013: 430438 . 